\title{
Human Embryonic Stem Cell Lines BJNhem 19 and 20 Fail to Differentiate Into Lung Lineage Specific Cells despite Induction through Guided Endodermal Differentiation
}

\author{
Srabani Kar, Garima Hore, Niladitya Sanyal and Ena Ray Banerjee* \\ Department of Zoology, University of Calcutta, 35, Ballygunge Circular Road, Kolkata- 700019, West Bengal, India \\ "Corresponding author: Ena Ray Banerjee, Associate Professor, Dept. of Zoology, University of Calcutta, 35, Ballygunge Circular Road, Kolkata- 700019, West \\ Bengal, India, Tel: 91-33-24615445 (Extn.275); Fax: 91-33-24614849; E-mail: enarb1@gmail.com
}

Received date: Jul 25, 2014; Accepted date: Nov06, 2014; Published date: Nov 10, 2014

Copyright: $\odot 2014 \mathrm{Kar}$ et al. This is an open-access article distributed under the terms of the Creative Commons Attribution License, which permits unrestricted use, distribution, and reproduction in any medium, provided the original author and source are credited.

\begin{abstract}
Regenerative Therapy's first and most critical requisite is the ex vivo synthesis of fully functional desired cells to replenish those lost by the body in a degenerative disease. The etiology of degeneration differs from disease to disease, likewise, strategies to regenerate lost tissue in its heterogeneousness, should also cater to the regeneration process and to customize the same, the researcher ought to devise strategies to repair or replace or regenerate in the appropriate spatio-temporal format. For example, in the inflammation-degeneration-induced pathophysiological situations of the respiratory epithelium, human embryonic stem cells have been used in a tissue engineering format to induce differentiation and amplification into the desired type of cell, in this case, the nonciliated squamous epithelial cells. To this end, we were given two human embryonic stem cell (hESC) lines and the main objective of our research work was to get BJNhem19 and BJNhem20 human embryonic stem cell (hESC) lines to differentiate into lung epithelial lineage-specific cells (i.e. alveolar epithelial type I and type II cells and clara cells) which are the key cells to degenerate in most degenerative lung ailments. This in order to generate a potentially unlimited supply of cells of the desired phenotype for use in a novel cell based therapy to repair lung injury. The strategy was to use guided endodermal differentiation by direct administration of one or more growth factors known to be involved in lung development in 2D cell cultures and characterize the cells for the desired markers. According to a tried strategy, the undifferentiated hESC were taken through embryoid body formation and then subjected to induction by defined growth factors in small airways growth medium and bronchiolar endothelial growth medium. Cells could not be grown feeder-free. Attempts to aid growth with combinations of extra cellular matrix plus defined medium (GeltrexR) as well as enriched media such as Matrigel and mTeSR1 did not yield satisfactory result. When grown on feeders also cell growth was again not optimum and practically none differentiated appreciably into the desired phenotype and maintained their pluripotent characters. After 5 days in induction media, they displayed fibroblast like features characterized by FACS. If cell lines are thus non-response to specific and guided endodermal induced differentiation, the obtained data is animportant information for cell repositories as numerous research labs screen various cell lines of embryonic origin with the specific aim to induce differentiation into a desired phenotype for functional translation into regenerative therapy. This study therefore fills an existing lacuna in available information regarding behavior of these two hESC available for work to the scientific world in several stem cell banks and shall prevent unnecessary and redundant further screening and save valuable resources.
\end{abstract}

Keywords: Human Embryonic Stem Cells; BJNhem; Pulmonary system; Lung epithelial lineage
Abbreviations:
Human Embryonic Stem Cells- hESC; Alveolar epithelial type I- AEI; Alveolar epithelial type II- AEII; Embryoid body- EB; Leukemia Inhibitory Factor-LIF; Stem Cell Factor-SCF; Colony Forming Assay- CFU; Clara cells-CC; Surfactant protein- SP; Aquaporin- AQP; Extracellular matrix- ECM

\section{Introduction}
The pulmonary system is composed of a variety of epithelial cell populations residing in distinct anatomical locations. Of these, the alveolar epithelial gas exchange surface consists of two cell types, the type I and type II pneumocytes, also known as alveolar epithelial type I and type II (AEI and AEII) cells, that comprise 95\% and 5\% respectively of the alveolar lining area [1]. The alveolar fluid balance is

regulated by AEI cells [2], structurally these are branched cells with extremely attenuated cytoplasm for gaseous exchange [3]. On the other hand AEII are cuboidal cells, and are located between AEI cells. These cells depict characteristics lamellar bodies and apical microvilli [3]. The main function of AEII cells include the creation and reuptake of pulmonary surfactant [4], regulation of alveolar fluid and synthesis of immunomodulatory proteins (SP-A, SP-D) important for host defense [5]. The columnar Clara cells [6] constitute the majority of the bronchiolar and terminal bronchiolar epithelia. Clara cells actively divided and differentiated to form ciliated cells, secrete glycosaminoglycans that are the major component of the extracellular matrix (ECM), and metabolized airborne toxins by cytochrome P-450 enzyme present in there smooth endoplasmic reticulum [7].

In many life threatening pulmonary diseases, such as acute lung injury, acute respiratory distress syndrome (ARDS), cystic fibrosis and idiopathic pulmonary fibrosis [8-10] endothelial cells along with AEI cells are the primary and initial sites of damage resulting in interstitial edema, increased ECM deposition such as collagen, laminin and 
Citation: Kar S, Hore G, Sanyal S, Banerjee ER (2014) Human Embryonic Stem Cell Lines BJNhem 19 and 20 Fail to Differentiate Into Lung Lineage Specific Cells despite Induction through Guided Endodermal Differentiation. J Tissue Sci Eng 6:144. doi: $10.4172 / 2157-7552.1000144$

Page 2 of 11

fibronectin in the lungs resulting in pulmonary fibrosis and loss of gaseous exchange surface. For lung injury repair, AEII cells and other lung progenitor cells may replace lost AEI cells to re - establish the thin barrier necessary for efficient gaseous - exchange in the alveolar milieu [11].

HESCs recently have been used as a potential source of cells for cellbased therapies in degenerative diseases when loss of function is apparent. Cell based tissue replacement therapy may have the potential to reinstate normal tissue structure and function $[12,13]$. For successful lung tissue replacement therapy a large number of lung lineage specific cells need to be engineered in vitro for transplantation. $\mathrm{Wnt} / \beta$-Catenin signaling has been identified as a key regulator of stem cell renewal with effect on cell proliferation and differentiation. In this Wnt signaling pathway $\beta$-Catenin increases in the nucleus and forms a complex with $t$ - cell factor (TCF) or lymphoid enhancer factor (LEF-1) transcription factor which are differentially modulated by Creb - Binding protein (CBP) and P300 co - activators. An increase in $\beta$ - Catenin or CBP mediated transcription by selectively inhibiting $\beta$ - Catenin or P300 mediated transcription maintains stem cell pluripotency, whereas blockade of $\beta$ - catenin/CBP signaling facilitates $\beta$-Catenin/P300 mediated transcription and cellular differentiation [14-16].

The main aim of our experiment was to differentiate hESCs into lung epithelial lineage - specific cells (AEI, AEII and Clara cells) and develop a cell based strategy to repair lung injury in a mouse model of IPF. Mouse ES and hES $\mathrm{H} 1$ cell lines have been previously demonstrated to have differentiated into AEII cells [17-20]. It is believed that AEII cells contribute to the repair of injured lungs by an initial limited proliferation followed by differentiation to AEI cells and Clara cells [21].

Our aim was to differentiate hES cells into lung epithelial lineagespecific cells [i.e., alveolar epithelial cells type I (AEI) and II (AEII) cells and Clara cells] and develop a cell-based strategy in order to repair lung injury in a mouse model of IPF. Previous work [22-30] has demonstrated differentiation steps to AEII cells from murine ES cells and the hES H1 cell line.

In this study we tried to differentiate hES BJNhem 19 and BJNhem 20 cells in culture into non - ciliated lung lineage specific cells and characterize them with intra - cellular and surface protein markers and subsequently characterize the cells morphologically [22]. The main idea was to check for down - regulation of pluripotent markers

Nanog, SSEA - 3 and SSEA - 4 and subsequently check for up regulation of lung lineage specific markers AQP - 5 and SP - C. In addition, we were also interested to find out if we could grow EBs from feeder free hESCs. Immunophenotyping was done using FACS and progenitor assessment was assessed by cellular colony formation (CFU).

We tried to grow the cells feeder free as published protocol of the originator lab [28-30] but could not obtain proper cell amplification that is good number of cells through multiple passages nor healthy cells (in stress-free phenotype). So we also attempted currently accepted combinations of extra cellular matrix plus defined medium (GeltrexR) as well as enriched media such as Matrigel and mTeSR1. But neither yielded suitably expanded cell numbers thereby indicating that the feeder-free condition is completely unsuitable for their growth. When grown on feeders also, cell growth was not optimum.
When moved to ultra-low attachment substratum with appropriate supporting factors for EB formation, uncharacteristic or atypical EB like bodies were grown. When moved to differentiation media, SAGM and BEGM, differentiation to required pulmonary-like phenotypes were not obtained (as compared to H7 [22]) with the BJNhem 19 and 20 cells lines continuing to express pluripotent markers nonresponsive to differentiation signals including transient heat shock.

Thus we conclude that the cells cannot be grown feeder free nor in supported media. Secondly, we attempted to induce respiratory differentiation by culturing cells in serum containing medium followed by SAGM or BEGM. We did not attempt to differentiate the cells via a mesendodermal precursor, via differentiation to foregut endoderm and finally to lung due to these aforementioned technical problems with the intrinsic nature of the cells (corroborated also by very poor clonogenic potential) indicating perhaps a functional disability of these particular huESC to undergo tissue differentiation per se or even to be maintained in culture without support. Based on these facts, we exclude the possibility that the cell lines could be useful as suitable research tools for induced endodermal differentiation.

The following sections shall elucidate these points by explicit data where we have used $\mathrm{H} 7$ as a positive control [22].

\section{Materials and Methods}

Media used: DMEM - HIMEDIA ,AL007S-500 ml, DMEM1(X) Gibco life technology,11960-044,500ml, Knockout TM DMEM F-12 1(x) - Gibco life technology $12660500 \mathrm{ml}$, RPMI1640 - HIMEDIA ALO28A $500 \mathrm{ml}$ MEM - HIMEDIA ALO47S $500 \mathrm{ml}$, IMDMHIMEDIA ALO70B- $500 \mathrm{ml}$, SAGM- Promo cell(GERMANY) C-21270 500ml, Fetal bovine serum (FBS) - HIMEDIA-RM1112 -500 $\mathrm{ml}$, Antibiotics antimycotic (PenStrep)- HIMEDIA A002A - $20 \mathrm{ml}$, Trypsin EDTA-1(x)- HIMEDIA -T001-100ml, L-Glutamine 200Mm HIMEDIA-TCL012-100ml Mitomycin -C 0454500-6, 5mg,CAYMAN Chemical company, Triidothyronine- CC- $4211 \mathrm{~F} .5 \mathrm{ml}$ lonza

Insuline - CC-4021F .5 ml lonza, BPE -CC-4009 2ml lonza, Retinoic acid - CC- 4085F .5 ml lonza, Epinephrine - CC-4221F .5 ml lonza, Transferrin- CC-4205F .5ml lonza, Hydrocortisone CC-4031F .5 ml lonza

\section{Ethics Statement}

All animal and animal related works was conducted according to the guidelines prescribed by Faculty Councils for Post Graduate Studies in Science, Technology and Engineering and Agriculture \& Veterinary Science (Ref. No. BEHR/1029/2304)

\section{Expansion of Human embryonic stem cells}

Human embryonic stem cell (hESCs) lines BJNhem19 and BJNhem 20 were obtained from JNCASR, India which is claimed to be karyotypically normal, sibling human ES cell lines representing the Indian ethnic background. These cells were derived from the inner cell mass (ICM) of grade III poor quality blastocysts that were not suitable for in vitro fertility treatment. Both the lines as claimed by JNCASR are pluripotent and have been extensively characterized and cultured continuously for over 250 passages [31-35].

The aforementioned ESC lines were grown on primary mouse embryonic feeder cells, for expansion and propagation in an undifferentiated state. The feeder cells were prepared from pregnant Balb C mice (13.5 days of gestation), these cells were then cultured in 
Citation: Kar S, Hore G, Sanyal S, Banerjee ER (2014) Human Embryonic Stem Cell Lines BJNhem 19 and 20 Fail to Differentiate Into Lung Lineage Specific Cells despite Induction through Guided Endodermal Differentiation. J Tissue Sci Eng 6: 144. doi: $10.4172 / 2157-7552.1000144$

Page 3 of 11

MEF conditioned media and then treated with Mitomycin - $\mathrm{c}$ to stop their differentiation. The MEF was cultured in Dulbecco's Modified Essential Medium (DMEM), 10\% fetal bovine serum (FBS) and 2mM - L Glutamine [36]. The hESCs were cultures in ES medium, comprised of Knock out (KO) DMEM supplemented with $20 \%$ Knock out serum replacement (KOSR), $1 \mathrm{mM}$ sodium pyruvate, $0.1 \mathrm{mM} 2 \beta$ Mercaptoethanol (ME), $0.1 \mathrm{mM}$ minimum essential media (MEM), $1 \%$ non-essential amino acids (NEEA), $1 \mathrm{mM} \mathrm{L}$-glutamine and 2 $\mathrm{ng} / \mathrm{ml}$ basic fibrobastic growth factor (bFGF). For the purposes of cell culture 6 well $10 \mathrm{~cm}^{2}$ tissue culture plates, coated with $0.1 \%$ gelatin were used, and all cultures were maintained in a humidified $5 \% \mathrm{CO}_{2}$ incubator at $37^{\circ} \mathrm{C}$. The protocol for induction of alveolar epithelial differentiation of hESCs was adapted from established methods (Figure 1) [16-20].

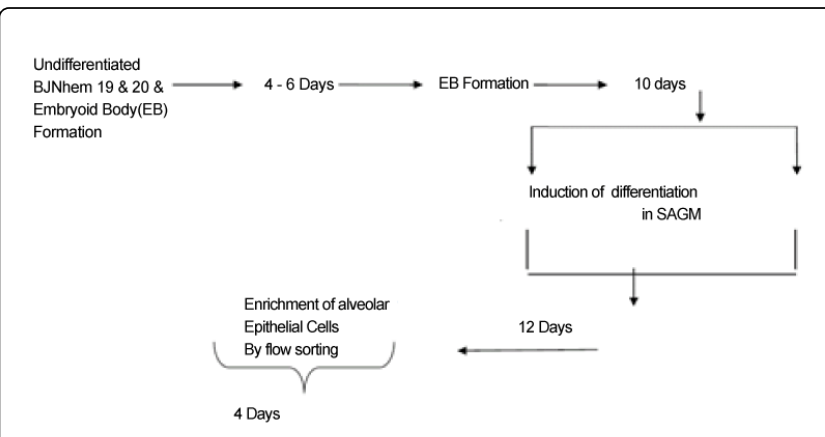

Figure 1: Outline of formation of EBs from Bjnhem20 cells and differentiation to alveolar epithelial cells in SAGM. Differentiation of BJNhem 19 and BJNhem 20 cells to lung epithelial cell - specific lineage. An outline of formation of EBs from BJNhem 19 and BJNhem 20 and differentiation to alveolar epithelial cells in SAGM and BEGM [22]

\section{Embryoid Body (EB) Formation}

hESCs were inspected on day 1 of passage and hESC culture colonies with well-defined boundaries and minimum differentiation were utilized. Dispase $1.2 \mathrm{U} / \mathrm{ml}$ dissolved in PBS (without magnesium and calcium) supplemented with $10 \%$ ES qualified FBS was used for detachment of undifferentiated hESCs at $37^{\circ} \mathrm{C}$. This treatment was carried out until all the hESC colonies were detached from the plates. The colonies were then washed off the plates using ES media without bFGF (washing done twice), and the cells were then resuspended in EB Medium (K.O. DMEM, 20\% KOSR, 20\% non - heat inactivated Fetal Calf serum, $1 \%$ NEAA, $1 \mathrm{mM} \mathrm{L}$ - Glutamine and $1 \mathrm{mM} 2 \beta-\mathrm{ME})$. Cells were subsequently transferred to ultra-low attachment 6 well plates and were grown for 4 days in suspension culture.

\section{Feeder Free culture of hESCs}

hESCs were initially cultured in hESC media as aforementioned. Once the cells became confluent these cells were passaged (about once a week) and subsequently cultured in high binding tissue culture plates in conditioned media prepared from MEF [incubation for 24 hours with mitotically inactivated MEFs] [31-35].

MEF were first isolated and cultured in MEF medium. The harvested cells were then treated with Mytomycin $-\mathrm{C}$ and $\sim 50,000$ cells were placed in MEF medium. The MEF media was exchanged with ES medium after 4-6 hours. This ES medium was collected on daily basis (24hrs as mentioned earlier) and supplemented with $4 \mathrm{ng} / \mathrm{ml}$ bFGF before feeding hESCs [37].

We also attempted currently accepted combinations of extra cellular matrix plus defined medium (GeltrexR) as well as enriched media such as Matrigel and mTeSR1 (data not presented for similar reasons of non-optimal growth).

\section{Expected differentiation/generation of non - ciliated pulmonary epithelial cells}

In order to generate non - ciliated pulmonary epithelial cells two different culture media were used. EBs after limited Dispase digestion was transferred to adherent culture in $0.1 \%$ gelatin coated tissue culture plates. One group of EBs were cultured in small airways growth medium (SAGM) for 12 days. [Small airways basal medium was procured from Promocell, bovine pituitary extract $30 \mu \mathrm{g} / \mathrm{ml}$, insulin $5 \mu \mathrm{g} / \mathrm{ml}$, hydrocortisone $0.5 \mu \mathrm{g} / \mathrm{ml}$, Gentamycin sulfate Amphotericin B $0.5 \mu \mathrm{g} / \mathrm{ml}$, bovine serum albumin $0.5 \mathrm{mg} / \mathrm{ml}$, transferrin $10 \mu \mathrm{g} / \mathrm{ml}$, epinephrine $0.5 \mu \mathrm{g} / \mathrm{ml}$ and recombinant human epidermal growth factor (rhEGF) $0.5 \mathrm{ng} / \mathrm{ml}$ ]. Cells were collected from 12 day culture in SAGM, cells were marked with surface expression markers SP-C+ and AQP-5 for FACS immunophenotyping.

The second group of EBs was cultured in bronchiolar epithelial growth media (BEGM) [Procured from Promocell], bovine pituitary extract $30 \mu \mathrm{g} / \mathrm{ml}$, insulin $5 \mu \mathrm{g} / \mathrm{ml}$, hydrocortisone $0.5 \mu \mathrm{g} / \mathrm{ml}$, Gentamycin sulfate - Amphotericin B $0.5 \mu \mathrm{g} / \mathrm{ml}$, retinoic acid 0.1 $\mathrm{ng} / \mathrm{ml}$, transferring $10 \mu \mathrm{g} / \mathrm{ml}$, triiodothyronine $6.5 \mathrm{ng} / \mathrm{ml}$, epinephrine $0.5 \mu \mathrm{g} / \mathrm{ml}$, rgEGF $0.5 \mathrm{ng} / \mathrm{ml}$. Media was refreshed every 2 days.

\section{Phenotypic Analysis of Cells}

Immunostaining was performed using specific antibodies. These specific antibodies were either conjugated with secondary antibodies or a secondary antibody had to be added to the primary antibody for staining. Phycoerythrin (PE) labeled Anti - human Nanog (BD Pharmigen, 560873), SSEA - 3 (Santa Cruz, 631: SC-21703) and SSEA - 4 (Santa Cruz, 813-70: SC - 21704) were used to determine if the hESCs cultured in SAGM and BEGM were still pluripotent or had differentiated into alveolar epithelial (AE) type I or Type II cells. In order to determine if the cells had differentiated in AE type I or II, we checked the cells for the following surface markers namely, Aquaporin 5 (AQP- 5, Santa Cruz Biotechnology, INC., G19: SC - 9890) and surfactant associated protein - C (SP-C, Santa Cruz Biotechnology, INC., C - 19: SC - 7705). Additionally, PerCp-Cy 5.5 Mouse anti Human CD105 (105) clone: 266, and PE Mouse Anti - Human CD73.clone AD2 (BD Pharmigen).

Based on the manufacturer's protocol, 106 cells were taken per sample in $50 \mu$ cell suspension in ice cold PBS (1x), $10^{5}$ events were recorded per sort for FACS analysis. For the FACS analysis cells were first stained with conjugated or non - conjugated antibodies and subsequently stained with a secondary antibody when needed. For staining purposes $5-10 \mu \mathrm{l}$ antibody was added to $10^{6}$ cells in suspension culture and placed on ice for 30 mins. After thorough washing, cells were fixed in $4 \%$ formaldehyde in PBS by vortexing and incubated at room temperature for $20 \mathrm{mins}$ followed by permeabilization in either $0.1 \%$ Tween -20 or $0.25 \%$ Triton X [25].

Cell suspension of $10^{6}$ cells per microfuge tube was prepared per sample and staining was carried out in a single step with a master mix of fluorochrome - conjugated monoclonal antibodies or in some cases 
Citation: Kar S, Hore G, Sanyal S, Banerjee ER (2014) Human Embryonic Stem Cell Lines BJNhem 19 and 20 Fail to Differentiate Into Lung Lineage Specific Cells despite Induction through Guided Endodermal Differentiation. J Tissue Sci Eng 6: 144. doi: $10.4172 / 2157-7552.1000144$

Page 4 of 11

where the primary antibody was not available in directly fluorochrome - conjugated form, in two steps of primary unlabelled antibody followed by cross reactive fluorochrome - conjugated specific secondary antibody at $4^{\circ} \mathrm{C}$ for 30 mins followed by rigorous wash (twice) in ice cold PBS. The antibody stained cell suspension was now analyzed using a BD FACS Verse ${ }^{\text {tm }}$ machine and data analyzed using software BD FACSuite version 1.0 or later [25].

\section{Cell Viability}

Viable cells were measured by trypan blue dye exclusion by light microscopy. Cell count and viability assays were performed using Tali image based cytometry manufactured by Invitrogen life technology (USA) and data analyzed using tali software version: 2.1anotographs taken with camera : 1.3 mega pixels, $4 \mathrm{x}$ objective, $4 \mathrm{x}$ or $16 \mathrm{x}$ digital zoom and Floid cell imaging system also manufactured by Invitrogen life technology (USA) and data analyzed using floid cell imaging station software, version: 3.1 (Catlog no : 4471136).

\section{CFU - C assay to determine progenitor cells}

To check for progenitor cells, CFU - C assay was performed using methylcellulose (Himedia), supplemented with 50ng of Stem cell factor (SCF) per $\mathrm{ml}$ in IMEM medium. 50,000 cells were plated in $35 \mathrm{~mm}$ plates and placed in incubator at $37^{\circ} \mathrm{C}$ and $5 \% \mathrm{CO} 2$ for 10 days. At the end of 10 days colony count was taken under microscope [data not shown] [25]. Cell count and viability assays were performed using Tali image based cytometry manufactured by Invitrogen life technology (USA) and data analyzed using tali software version: 2.1 anotographs taken with camera : 1.3 mega pixels, $4 \mathrm{x}$ objective, $4 \mathrm{x}$ or 16x digital zoom and Floid cell imaging system also manufactured by Invitrogen life technology (USA) and data analyzed using floid cell imaging station software, version: 3.1 (Catlog no : 4471136).

\section{Results}

\section{Failure of hESC BJNhem 19 and 20 to grow on feeder-free cultures versus $\mathrm{H} 7$ which did}

Cells from the hES cell line $\mathrm{H} 7$ were differentiated in vitro into three lung lineage-specific epithelial cells: AEI cells, AEII cells, and Clara cells as described below. These cells expressed, both intracellularly and on their surface, characteristic marker proteins, detected byfluorescence-activated cell sorting (FACS) and immunofluorescence (IF) microscopy, the mRNA for which were also concomitantly over-expressed as detected by quantitative real-time PCR (qPCR). The protocol for differentiation of pluripotent undifferentiated colonies of $\mathrm{H} 7 \mathrm{hES}$ cells into the lung epithelial cellspecific lineages is shown in Figure 1. This is clearly evident from Figures 2-4 where very sparse growth of cells is observed as opposed to Figure 1 that clearly shows a rich growth of cells in a with feeder and a feeder free condition.

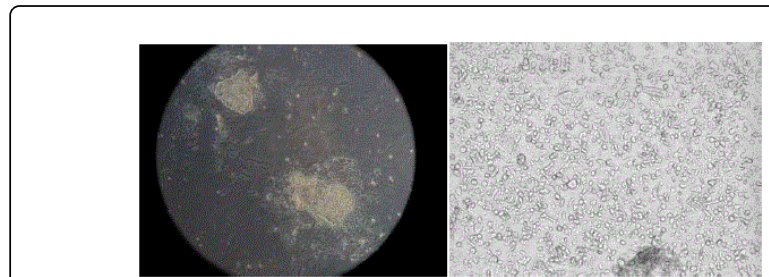

Figure 2: With feeder and without feeder (conditioned media) culture of H7. Day 1-6 in adherent culture as described [22]. Photographs taken with a Bright field phase contrast microscope: model no Olympus BX41, camera model no Olympus DP20 at 10X and (photo inset) 40X magnification.

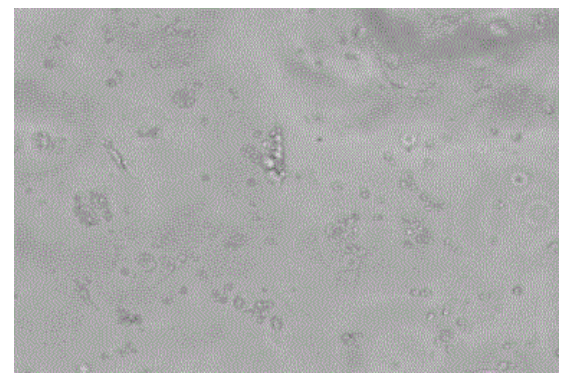

Figure 3: Feeder-free culture of undifferentiated huESC (BJNhem 19 \& 20) in conditioned medium. Undifferentiated hES cells on mitomycin-c inactivated MEF feeder-conditioned media for 4-6 days followed by formation of EBs. Details of microscopy are as follows: Photographs were taken at 40X magnification using a Leica DMIL microscope (Leica Microsystems GmbH, Germany) and a Zeiss ApoTome (Carl Zeiss Microimaging GmbH, Germany). Photographs were taken with a Zeiss Axiovert 200M microscope and Axiocam MRm and merged using Axiovision 4.6 software.

\section{Sequential but statistically non-significant downregulation of pluripotent markers}

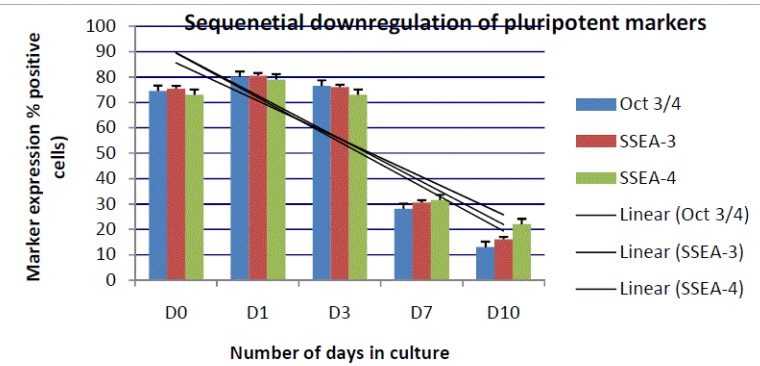

Figure 4: Oct3/4, SSEA-3, SSEA-4 for identification of differentiation stage of the cells in culture.

The percentage of positive cells is shown as mean \pm SEM ( $n=3$ independent experiments with flow data collected in triplicate). BD Accuri C6 FACS machine was used to acquire data which was analyzed with Accuri C6 software. Trendline shows a progressive 
Citation: Kar S, Hore G, Sanyal S, Banerjee ER (2014) Human Embryonic Stem Cell Lines BJNhem 19 and 20 Fail to Differentiate Into Lung Lineage Specific Cells despite Induction through Guided Endodermal Differentiation. J Tissue Sci Eng 6: 144. doi: $10.4172 / 2157-7552.1000144$

Page 5 of 11

down modulation of pluripotent markers which nevertheless seem to settle to more than $40 \%$ at around 5-6 days of culture except for Oct $3 / 4$.

\section{EB formation}

The EBs failed to differentiate into AE type I, type II and clara cells in SAGM medium and BEGM medium respectively (Figure 5A-5C).

FACs analysis yielded negative for the aforementioned desired cell lines. In addition, both BJNhem 19 and BJNhem 20 failed to sustain themselves in feeder free cultures.

A.

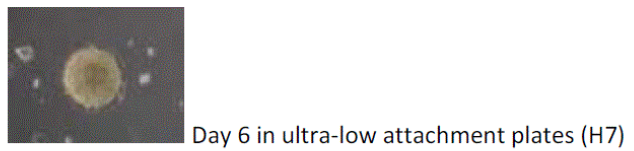

B.

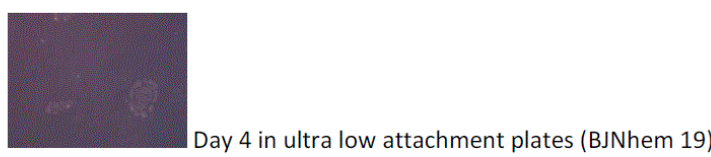

C.

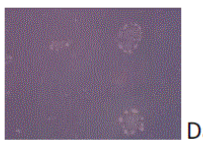

Day 6 in ultra low attachment plates (BJNhem 19)

Figure 5A-C: EB formation in ultra-low attachment plates. EBs were formed in suspension culture overnight after aggregation. day 4 EBs were cultured in ultra-low attachment plates for 10 days and then transferred to gelatin-coated plates and cultured with either SAGM or BEGM. Photographs were taken at $40 \mathrm{X}$ magnification using a Leica DMIL microscope (Leica Microsystems GmbH, Germany) and a Zeiss ApoTome (Carl Zeiss Microimaging GmbH, Germany).Photographs were taken with a Zeiss Axiovert 200M microscope and Axiocam MRm and merged using Axiovision 4.6 software.

\section{Differentiation in induction medium BJNhem 19 (Figure 6)}

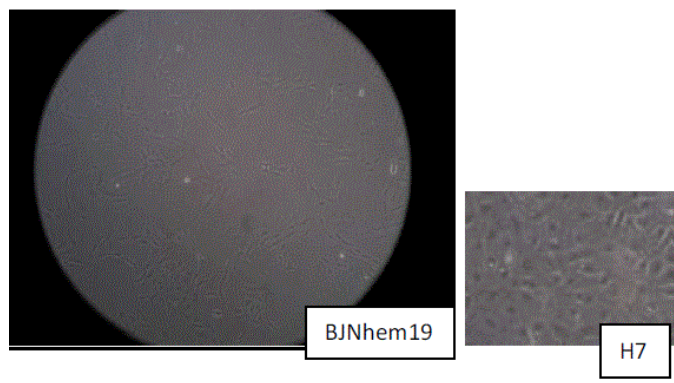

Figure 6: Following the formation of EB-like 3D structures, they were transferred to pro attachment tissue culture treated plates and subjected to various differentiation induction media.

SAGM-induced differentiation yielded a fibroblast-like phenotype where cell growth was rapid. Picture taken by Plympus BX41 (Japan), photo taken by Olympus cool camera (model no. DP20) and image processed by MIcroPublisher 5.0 RTV 5.0 X 106 pixels in frame rate 25 fps. Fibroblast like phenotype after 10 days of culture in SAGM (BJNhem20).

\section{Differentiation in induction medium BJNhem 20 (Figure 7 and 8)}

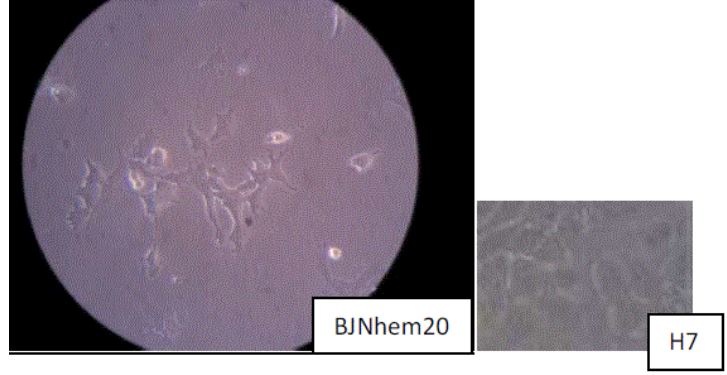

Figure 7: Cuboidal phenotype after 10 days of culture in BEGM (BJNhem20). Photo taken in Floid Imaging station manufactured by Invitrogen, Life Technologies, CA, USA.

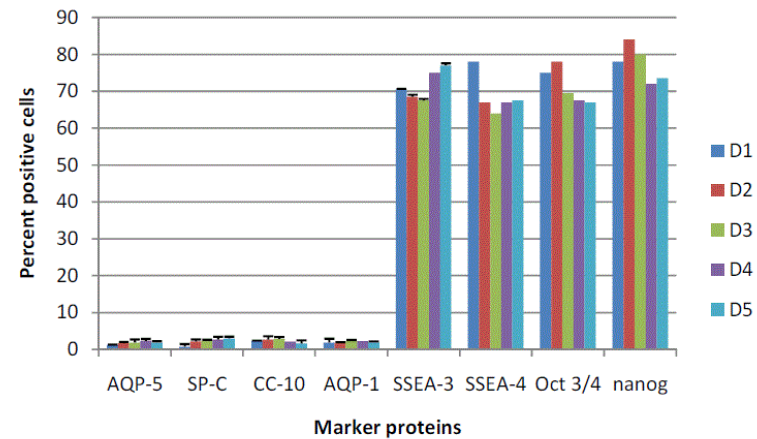

Figure 8: Expression of surface and intra-cellular markers following differentiation in induction medium SAGM in tissue culture treated plates: EB were transferred to tissue culture treated plates and grown in induction medium composed of SABM; bovine pituitary extract (BPE) $30 \mu \mathrm{g} / \mathrm{ml}$, insulin $5 \mu \mathrm{g} / \mathrm{ml}$, hydrocortisone (HC) $0.5 \mu \mathrm{g} / \mathrm{ml}$, gentamycin sulfate-amphotericin B $0.5 \mu \mathrm{g} / \mathrm{ml}$, bovine serum albumin (BSA) $0.5 \mathrm{mg} / \mathrm{ml}$, transferrin $10 \mu \mathrm{g} / \mathrm{ml}$, epinephrine $0.5 \mu \mathrm{g} / \mathrm{ml}$, and recombinant human epidermal growth factor (rhEGF) $0.5 \mathrm{ng} / \mathrm{ml}$. Cell count taken using Tali ${ }^{\mathrm{mm}}$ Image based cytometer manufactured by Invitrogen, Life Technologies, USA. Photo taken by camera 1.3 megapixels, $4 \mathrm{X}$ objective, $4 \mathrm{X}$ or $16 \mathrm{X}$ digital zoom.

\section{Additional stimuli for induction}

HuESC were treated to brief exposure to $67^{\circ} \mathrm{C}$ for 10 minutes to induce heat shock. The cellular response to heat shock includes the transcriptional up-regulation of genes encoding heat shock proteins (HSPs) as part of the cell's internal repair mechanism [31] (Figure 9). They are also called stress-proteins [32] and respond to heat, cold and oxygen deprivation by activating several cascade pathways [33-35]. Transient heat shock- exposure of the cells to $67^{\circ} \mathrm{C}$ for $30 \mathrm{~min}$ 
Citation: Kar S, Hore G, Sanyal S, Banerjee ER (2014) Human Embryonic Stem Cell Lines BJNhem 19 and 20 Fail to Differentiate Into Lung Lineage Specific Cells despite Induction through Guided Endodermal Differentiation. J Tissue Sci Eng 6: 144. doi: $10.4172 / 2157-7552.1000144$

Page 6 of 11

followed by transfer to $22^{\circ} \mathrm{C}$ in SAGM results in a mixed phenotype of cells [31-35].

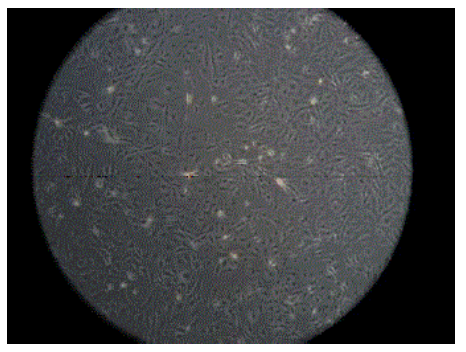

Figure 9: HuESC were treated to brief exposure to $670 \mathrm{C}$ for 10 minutes to induce heat shock. The cellular response to heat shock includes the transcriptional up-regulation of genes encoding heat shock proteins (HSPs) as part of the cell's internal repair mechanism [30]. They are also called stress-proteins [31] and respond to heat, cold and oxygen deprivation by activating several cascade pathways. [32-35] Mixed phenotype in SAGM with transient hear shock (BJnhem20)

\section{Characterization of differentiated cell in SAGM (Figure 10} A-H)

Flow cytometry of surface and intracellular markers of cells differentiated in induction medium (SAGM) for 10 days show very little change to the desired phenotype namely lung lineage specific phenotype (there was hardly specific pulmonary ciliated squamous and cuboidal epithelial marker expression). The predominant phenotype obtained was that of fibroblasts, not specific to the lung, and they were some CD45-and some actually CD45+. AEII specific SP-C and AQP-1 and AEI specific AQP-5 expression was $<1 \%$ and considered negligible.

Figure 10A: Down-modulation of pluripotent specific markers and sequential upregulation of lineage specific (in this case pulomonary lineage) marker expression (intra-cellular protein) was expected. Cells were therefore permeabilized using permeabilization kit (BD biosciences, USA) and stained with specific antibodies against isotype matched control antibodies. With the lineage specific markers irrelevant markes were also used in different cell samples from the same experimental pool. Dulicate samples of cells were stained per experiment and overall experimental output from three independent experiments is presented here. BD Accuri C6 was used to acquire data which was analyzed using BD Accuri C6 software. Cells were stained with specific antibodies (detailed in Materials and Methods section). This figure represents Fibroblast marker expressing cells where the scattergram represents: $\mathrm{X}$-axis CD45-PerCp; Y-axis CD10-FITC (CD45+CD10+ cell population is $31 \%$ and is found in the upper right quadrant and CD45-CD10+ cell population is $66 \%$ and is found in the lower right LR quadrant). Figure 10B: Embryoid bodies were transferred into induction medium (SAGM) and cultured in adherent culture for 48 hours and over the next three days (in total 5 days) for optimal growth. Down-modulation of pluripotent specific markers and sequential upregulation of lineage specific (in this case pulmonary lineage) marker expression (intra-cellular protein) was expected. Cells were therefore permeabilized using permeabilization kit (BD biosciences, USA) and stained with specific antibodies against isotype matched control antibodies. With the lineage specific markers irrelevant markers were also used in different cell samples from the same experimental pool.

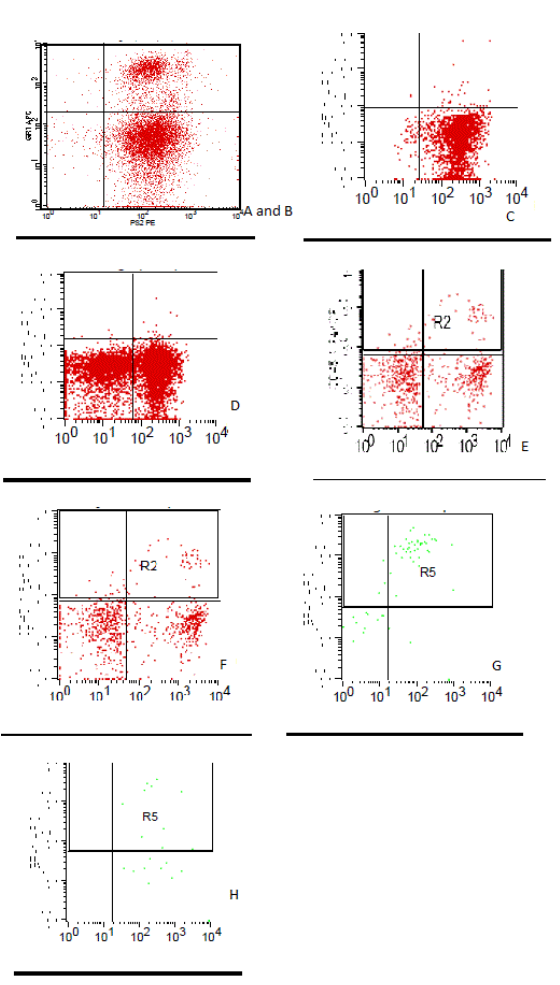

Figure 10A-H: Embryoid bodies were transferred into induction medium (SAGM) and cultured in adherent culture for 48 hours and over the next three days (in total 5 days) for optimal growth.

Duplicate samples of cells were stained per experiment and overall experimental outputs from three independent experiments are presented here. BD Accuri $\mathrm{C} 6$ was used to acquire data which was analyzed using BD Accuri C6 software. Cells were stained with specific antibodies (detailed in Materials and Methods section). This figure represents Fibroblast marker expressing cells where the scattergram represents: X-axis is CD45- gated and Y-axis is SP-C-FITC. Lung lineage specific lineages (SP-C+cells which are the Type II pneumocytes or alveolar epithelial cells is less than 2\% (UL quadrant). Figure 10C: Embryoid bodies were transferred into induction medium (SAGM) and cultured in adherent culture for 48 hours and over the next three days (in total 5 days) for optimal growth. Down-modulation of pluripotent specific markers and sequential upregulation of lineage specific (in this case pulomonary lineage) marker expression (intracellular protein) was expected. Cells were therefore permeabilized using permeabilization kit (BD biosciences, USA) and stained with specific antibodies against isotype matched control antibodies. With the lineage specific markers irrelevant markers were also used in different cell samples from the same experimental pool. Duplicate samples of cells were stained per experiment and overall experimental output from three independent experiments is presented here. $\mathrm{BD}$ Accuri C6 was used to acquire data which was analyzed using BD Accuri C6 software. Cells were stained with specific antibodies (detailed in Materials and Methods section). This figure represents 
Citation: Kar S, Hore G, Sanyal S, Banerjee ER (2014) Human Embryonic Stem Cell Lines BJNhem 19 and 20 Fail to Differentiate Into Lung Lineage Specific Cells despite Induction through Guided Endodermal Differentiation. J Tissue Sci Eng 6: 144. doi: $10.4172 / 2157-7552.1000144$

Page 7 of 11

Fibroblast marker expressing cells where the scattergram represents: $\mathrm{X}$ - axis CD45-PerCp. Hematopoietic lineage (pan-hematopoietic lineage negative). Y-axis is an irrelevant isotype matched antibody to rule our non-hematopoietic lineages. Figure 10D: Embryoid bodies were transfer red into induction medium (SAGM) and cultur ed in adherent culture for 48 hours and over the next three days (in total 5 days) for optimal growth. Down-modulation of pluripotent specific markers and sequential upregulation of lineage specific (in this case pulomonary lineage) marker expression (intra-cellular protein) was expected. Cells were therefore permeabilized using permeabilizattion kit (BD biosciences, USA) and stained with specific antibodies against isotype matched control antibodies. With the lineage specific markers irrelevant markers were also used in different cell samples from the same experimental pool. Duplicate samples of cells were stained per experiment and over all experimental output from three independent experiments are presented here. BD Accuri C6 was used to acquire data which was analyzed using BD Accuri C6 software. Cells were stained with specific antibodies (detailed in Materials and Methods section). This figure represents Fibroblast marker expressing cells where the scattergram represents: X- Axis: CD81+CD47+ cell population is represented in the LR quadrant and is $67.5 \%$ of the sample. These are fibroblast specific markers. LL quadrant represents Annexin V positive cells and is excluded. Figure 10E: Embryoid bodies were transferred into induction medium (SAGM) and culture $d$ in adherent culture for 48 hours and over the next three days (in total 5 days) for optimal growth. Down-modulation of pluripotent specific markers and sequential upregulation of lineage specific (in this case pulmonary lineage) marker expression (intra-cellular protein) was expected. Cells were therefore permeabilized using permeabilizattion kit (BD biosciences, USA) and stained with specific antibodies against isotype matched control antibodies. With the lineage specific markers irrelevant markers were also used in different cell samples from the same experimental pool. Duplicate samples of cells were stained per experiment and over all experimental output from three independent experiments are presented here. BD Accuri C6 was used to acquire data which was analyzed using BD Accuri C6 software. Cells were stained with specific antibodies (detailed in Materials and Methods section). This figure represents Fibroblast marker expressing cells where the scattergram represents: R2 is CD45- gated SP-C+ cell population and represents non-hematopoietic cells of Type II lineage and is a very insignificant population (UR 1.34\%). Figure 10F: Embryoid bodies were transferred into induction medium (SAGM) and cultured in adherent culture for 48 hours and over the next three days (in total 5 days) for optimal growth. Down-modulation of pluripotent specific markers and sequential upregulation of lineage specific (in this case pulmonary lineage) marker expression (intracellular protein) was expected. Cells were therefore permeabilized using permeabilization kit (BD biosciences, USA) and stained with specific antibodies against isotype matched control antibodies. With the lineage specific markers irrelevant markers were also used in different cell samples from the same experimental pool. Duplicate samples of cells were stained per experiment and overall experimental output from three independent experiments is presented here. BD Accuri C6 was used to acquire data which was analyzed using BD Accuri C6 software. Cells were stained with specific antibodies (detailed in Materials and Methods section). This figure represents Fibroblast marker expressing cells where the scattergram represents: $\mathrm{R} 2$ is CD45- gated AQP-1+ cell population and represents nonhematopoietic cells of Type I lineage and is also a very insignificant population (UR 0.67\%). Figure 10G: Embryoid bodies were transferred into induction medium (SAGM) and cultured in adherent culture for
48 hours and over the next three days (in total 5 days) for optimal growth. Down-modulation of pluripotent specific markers and sequential upregulation of lineage specific (in this case pulmonary lineage) marker expression (intra-cellular protein) was expected. Cells were therefore permeabilized using permeabilization kit (BD biosciences, USA) and stained with specific antibodies against isotype matched control antibodies. With the lineage specific markers irrelevant markers were also used in different cell samples from the same experimental pool. Duplicate samples of cells were stained per experiment and overall experimental output from three independent experiments is presented here. BD Accuri C6 was used to acquire data which was analyzed using BD Accuri C6 software. Cells were stained with specific antibodies (detailed in Materials and Methods section). This figure represents Fibroblast marker expressing cells where the scattergram represents: R5 is CD45- gated AQP-5+ cell population and represents non-hematopoietic cells of Type II lineage and is also a very insignificant population (UR $0.67 \%$ ). This is a confirmatory characterization of Type II alveolar epithelial cells. Figure $10 \mathrm{H}$ : Embryoid bodies were transferred into induction medium (SAGM) and cultured in adherent culture for 48 hours and over the next three days (in total 5 days) for optimal growth. Down-modulation of pluripotent specific markers and sequential upregulation of lineage specific (in this case pulmonary lineage) marker expression (intracellular protein) was expected. Cells were therefore permeabilized using permeabilization kit (BD biosciences, USA) and stained with specific antibodies against isotype matched control antibodies. With the lineage specific markers irrelevant markers were also used in different cell samples from the same experimental pool. Duplicate samples of cells were stained per experiment and overall experimental output from three independent experiments is presented here. BD Accuri C6 was used to acquire data which was analyzed using BD Accuri C6 software. Cells were stained with specific antibodies (detailed in Materials and Methods section). This figure represents Fibroblast marker expressing cells where the scattergram represents: R5 is CD45- gated TTF-1+ is $0.31 \%$ and represents lung lineage specific cell population (characteristic of embryonic lung) and is represented in quadrant UR of the scattergram.

\section{Clonogenic potential of hES cells declines with differentiation}

In tandem with characterization the identity of the cells, we proceeded to quantify clonogenic potential of the progenitors as they progress into differentiation steps to become lung lineage specific cells. As the ESC was taken through the various tissue engineering setups towards lineage specific differentiation, clonogenicity of the cells is an important yardstick for measurement of functionality (Figure 11).

\section{Discussion}

This study describes a novel strategy that have been used with spectacular success for differentiating hES cells into endodermal lung lineage-specific cells using human ESCH7 from WiCells, WI, USA [8]. Alteration of the differentiation medium strikingly modified the pathway of differentiation of EBs into different cell types as we found using the H7 ESC. Culture of EBs in a commercially available medium used for maintaining primary culture of mature pulmonary alveolar cells SAGM (excluding tri-iodothyronin and retinoic acid) promoted a predominantly AEII cell phenotype there. In contrast, culture in a commercially available BEGM (with tri-iodothyronin and retinoic acid 
Citation: Kar S, Hore G, Sanyal S, Banerjee ER (2014) Human Embryonic Stem Cell Lines BJNhem 19 and 20 Fail to Differentiate Into Lung Lineage Specific Cells despite Induction through Guided Endodermal Differentiation. J Tissue Sci Eng 6: 144. doi: $10.4172 / 2157-7552.1000144$

Page 8 of 11

but without BSA) promoted differentiation to predominantly bronchiolar alveolar cell (i.e., Clara cell) phenotype.

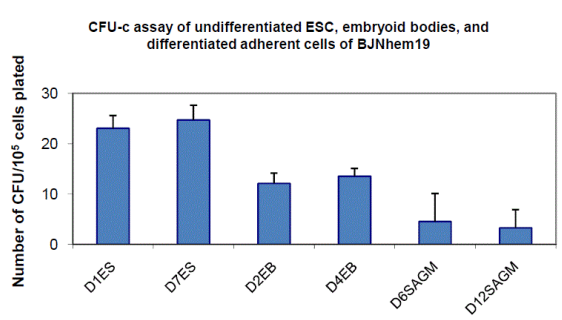

Figure 11A: CFU-c (colony forming assay counts at day 7 of BJNhem20 in methyl cellulose with SCF-1 in IMDMundifferentiated ES, EBs and differentiated in SAGM (BJNhem 19). Details of procedure outlined in Materials and Methods section. The presented graphs represent number of colonies formed per). 1 million cells plated in the semi-solid 3D matrix of methyl-cellulose supplemented with IMDM and SCF. CFU-c at day 7 of BJNhem 20 in methyl cellulose with SCF-1 in IMDM- undifferentiated ES, EBs and differentiated in SAGM (BJNhem 20). Y-axis represents number of days in culture in varying media and differnetial attachment templates.

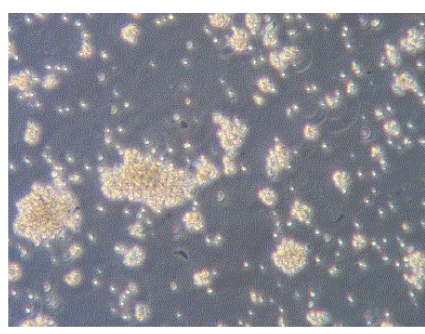

Figure 11B: Photograph of colonies counted and data presented for Fig.10A, was taken using Bright field phase contrast microscope: model no Olympus BX41, camera model no Olympus DP20 at 40X magnification)

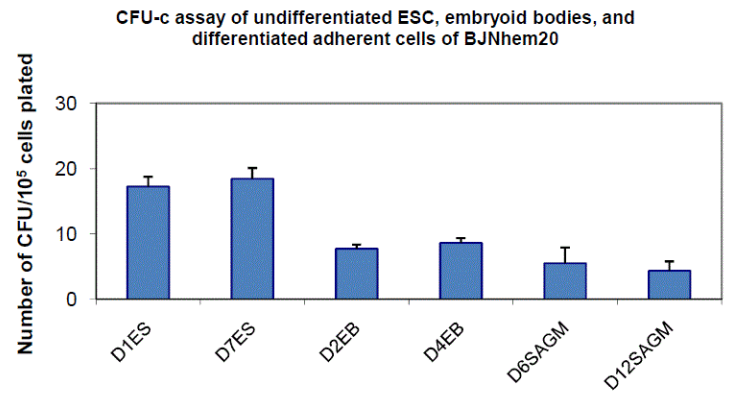

Figure 11C: Picture at 100X magnification of colony forming units from undifferentiated ES, EBs and differentiated in SAGM (BJNhem 20) A. Positive control (H7 [22 unpublished data], B. BJNhem 20.

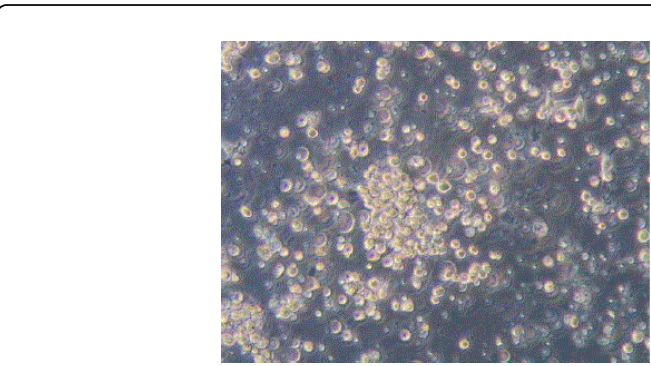

Figure 11D: Photograph of colonies counted and data presented for Figure $11 \mathrm{~A}$, was taken using Bright field phase contrast microscope: model no Olympus BX41, camera model no Olympus DP20 at 40X magnification)

Lung lineage-specific cell differentiation was achieved in a relatively shorter span of time (22 days) in contrast to other reported lung lineage culture conditions [22-30]. These culture media, normally used to maintain and grow mature cells, could successfully induce differentiation of pluripotent embryonic stem cells into three types of mature lung lineage-specific non-ciliated cells. Whereas AEI cells and AEII cells are found in the alveolar areas, Clara cells are found in terminal bronchioles. This aforementioned study demonstrated that from the same clonal population of undifferentiated hES cells, tissue engineering can be used to skew differentiation into one or another type of functionally competent mature cells.

In line with this same tried and tested hypothesis [22], we embarked upon inducing directed and guided differentiation of two cell lines BJNhem 19 and 20 generated in a JNCSAR lab [30-34]. We used similar coaxing of the ESC as in our work with $\mathrm{H} 7$ [22]. An important reason for our working with these cells was the originator's claim that the cells could grow in feeder-free conditions as while working with H7 (from WiCells, Madison, WI) the huESC could not be grown feeder-free which proved problematic during xenograft transplantation in a mouse model of bleomycin-induced lung fibrosis, an injury model where stem cells are important mobilizers from tissue niche of the adult lung and bone marrow and may be used as an effective strategy to induce regeneration. Lineage differentiated cells could also help reconstruct the lung from inside out by replacing damaged or infected cells in degenerative lung disease. To this end, we tried to condition the media following the published protocol of the originator lab and culture the same for amplification before subjecting to tissue engineering. Feeder-free growth failed completely as we got very poor yield and unhappy cells with stress fibres (Data not shown) which prompted us to shift to using irradiated mouse feeder cells (MEF) for culturing the huESCells in an undifferentiated state for propagation and use in the subsequent differentiation protocols as was our primary goal.

Despite various tested efforts such as combinations of ECM proteins coating the substratum for attachment, use of defined medium with growth factors (following the manufacturer's protocol) (GeltrexR) as well as enriched media such as Matrigel and mTeSR1. But none yielded healthy growth of cells nor is optimally expanded cell numbers thereby indicating that there is a problem with the cells feeder-free condition completely unsuitable for their growth. When grown on feeders also, cell growth was not optimumand additional protocol to induce differentiation through tissue engineering techniques, lung lineage specific cellular differentiation in both human 
Citation: Kar S, Hore G, Sanyal S, Banerjee ER (2014) Human Embryonic Stem Cell Lines BJNhem 19 and 20 Fail to Differentiate Into Lung Lineage Specific Cells despite Induction through Guided Endodermal Differentiation. J Tissue Sci Eng 6: 144. doi: $10.4172 / 2157-7552.1000144$

Page 9 of 11

ESC lines BJNhem 19 and 20 were found to be unsatisfactory. Repeated passages, fresh sub-cultures, feeder-conditioned cultures and on-feeder-cultures, with and without embryoid body formation failed to induce differentiation of these hES in culture into non-ciliated lung lineage-specific cells with intracellular and surface protein markers and morphology characteristic of AEI cells, AEII cells, and Clara cells.

According to the originator lab's published papers, BJNhem 20 cells were seen to divide spontaneously into cardiomyocytes in later passages and their differentiation efficiency was increased with the induction of DMSO. [37]. Venu and team observed that only about 5\% of early passage EBs showed spontaneous appearance of beating cardiomyocytes but in subsequent later passages this percentage increased to $45.5 \%$ (p101), 58.3\% (p115), and 62.5\% (p135) of beating cardiomyocytes. In order to validate these beating cardiomyocytes these cells were checked for cardiac progenitor marker Tbx 5 and cardiomyocytes marker a - actinin. Although, only a subset of cultured cells expressed Tbx5, all cells analyzed showed $\alpha$ - actinin. These results as per the authors confirm that the BJNhem 20 cell line is capable of differentiating into cardiomyocytes. However, it might be noted that the BJNhem19 cell line did not show any spontaneous differentiation in to cardiomyocytes ([36-40].

\section{Our observations show}

Neither huESC (BJNhem 19 nor 20) could grow independently of feeder as claimed in the literature from the originator lab nor in other defined media enriched with growth factors expected to promote appreciable growth in healthy condition of the cells to continue in undifferentiated state in the presence of hub a FGF.

Inactivated mouse embryonic fibroblast feeders can support their growth but after 10 passages, a pronounced downturn in pluripotent marker expression is observed. This is not desirable for a cell line which should sustain comparable growth and phenotypic character for at least $35-40$ passages.

Embryoid bodies looked atypical following culture in high attachment plates in EB media on day 3 of culture but did not show significant expression of intracellular markers for lineage commitment.

Spontaneous cardiac differentiation was not tested but upon induction under influence of various differentiation media (SAGM and BEGM) with defined components, insignificant number of cells (less than 2\%) showed any endodermal lineage specific differentiation, if at all, and a predominantly fibroblast like lineage was detected which is deemed unsuitable for regenerative therapy.

These differentiated cells showed pronounced fibrin-like protein synthesis and deposition upon in vitro culture on tissue culture treated high attachment plates.

With pluripotent marker expression progressively downmodulating only after 72 hours of culture, formation of atypical EBs and expression of pulmonary lineage specific markers in less than $1-2 \%$ of cells (of non-hematopoietic cells), clonogenic potential was found to be low (15-20\%).

While in maintenance media (for undifferentiated cells) pluripotent markers down modulated and was found to have become stable around $50 \%$ by 72 hours of culture, when EBs were transferred to induction media with defined growth factors, upto $70 \%$ of the cells were found to be positive for pluripotent markers such as nanog, oct $3 / 4$, SSEA-3 and 4 indicating a lack of response to differentiation stimuli. Despite further stimulus to induce differentiation by transient heat shock, this non-response could not be reversed.

This brings up the important question of the suitability of certain cells versus others in the work for induced differentiation into a certain cell type of a certain germinal lineage. The cells showed fibroblast like phenotype upon induced guided differentiation with defined growth factors. The cells showed a high level of pluripotent markers despite being cultured in a pointedly differentiation inducing medium. The cells showed low CFU-c or clonogenic potential and the cells show resistance to differentiation into the desired cell phenotype although other ESC lines such as H1, H7, KCL002-WT4, Shef- 6 and others do [41-62].

To form a large diffusible interface capable of conducting respiratory gases to and from the circulation, the lung must undergo extensive cell proliferation, branching morphogenesis, and alveolar saccule formation, to generate sufficient surface area. In addition, the cells must differentiate into at least 40 distinct lung cell lineages. Specific transcriptional factors, peptide growth factor receptormediated signaling pathways, extracellular matrix components, and integrin-signaling pathways interact to direct lung morphogenesis and lung cell lineage differentiation [59]. Some cell lines may be better adapted or in more precise terms, possess a predisposition to responding to becoming some germinal layer-derived cells versus others and therefore while undertaking tissue engineering exercises with follow-up functional assays on the functional attributes of an engineered cell, these factors need be documented. Stem cell banks may participate in the formation and collation of a comprehensive database enumerating these attributes and systems biology analysis of their epigenetic tendencies may reveal patterns and predictive value of such signals that may save valuable resources when attempts are made to engineer ESC into specific cell types.

\section{Acknowledgements}

The authors wish to thank Department of Biotechnology, Govt. of India for funding the project that resulted in this work (BT/ PR133331/MED/31/82/2009 dt 3/8/2013). The fellowships of SK, GH and NS were provided by DBT.

\section{References}

1. Gomperts BN, Strieter RM (2007) Stem cells and chronic lung disease. Annu Rev Med 58: 285-298.

2. Dahlin K, Mager EM, Allen L, Tigue Z, Goodglick L, et al. (2004) Identification of genes differentially expressed in rat alveolar type I cells. Am J Respir Cell Mol Biol 31: 309-316.

3. Hoppeler H, Lindstedt SL, Claassen H, Taylor CR, Mathieu O, et al. (1984) Scaling mitochondrial volume in heart to body mass. Respir Physiol 55: 131-137.

4. Laffon M, Jayr C, Barbry P, Wang Y, Folkesson HG, et al. (2002) Lidocaine induces a reversible decrease in alveolar epithelial fluid clearance in rats. Anesthesiology 96: 392-399.

5. Evans MJ, Cabral-Anderson LJ, Freeman G (1978) Role of the Clara cell in renewal of the bronchiolar epithelium. Lab Invest 38: 648-653.

6. Tai G, Polak JM, Bishop AE, Christodoulou I, Buttery LD (2004) Differentiation of osteoblasts from murine embryonic stem cells by overexpression of the transcriptional factor osterix. Tissue Eng 10: 1456-1466.

7. Kasper M, Schöbl R, Haroske G, Fischer R, Neubert F, et al. (1996) Distribution of von Willebrand factor in capillary endothelial cells of rat lungs with pulmonary fibrosis.Exp Toxicol Pathol 48:283-288. 
Citation: Kar S, Hore G, Sanyal S, Banerjee ER (2014) Human Embryonic Stem Cell Lines BJNhem 19 and 20 Fail to Differentiate Into Lung Lineage Specific Cells despite Induction through Guided Endodermal Differentiation. J Tissue Sci Eng 6: 144. doi: $10.4172 / 2157-7552.1000144$

Page 10 of 11

8. Kasper M, Haroske G (1996) Alterations in the alveolar epithelium after injury leading to pulmonary fibrosis. Histol Histopathol 11: 463-483.

9. Kuwano K, Kunitake R, Maeyama T, Hagimoto N, Kawasaki M, et al. (2001) Attenuation of bleomycin-induced pneumopathy in mice by a caspase inhibitor. Am J Physiol Lung Cell Mol Physiol 280: L316-325.

10. Uhal BD (1997) Cell cycle kinetics in the alveolar epithelium. Am J Physiol 272: L1031-1045.

11. Stripp BR, Reynolds SD (2005) Bioengineered lung epithelium: implications for basic and applied studies in lung tissue regeneration. Am J Respir Cell Mol Biol 32: 85-86.

12. Filippatos GS, Hughes WF, Qiao R, Sznajder JI, Uhal BD (1997) Mechanisms of liquid flux across pulmonary alveolar epithelial cell monolayers. In Vitro Cell Dev Biol Anim 33: 195-200.

13. Plopper CG, Mango GW, Hatch GE, Wong VJ, Toskala E, et al. (2006) Elevation of susceptibility to ozone-induced acute tracheobronchial injury in transgenic mice deficient in Clara cell secretory protein.Toxicol Appl Pharmacol 213:74-85.

14. Yoshikawa S, Miyahara T, Reynolds SD, Stripp BR, Anghelescu M, et al (2005) Clara cell secretory protein and phospholipase A2 activity modulate acute ventilator-induced lung injury in mice. J Appl Physiol (1985) 98: 1264-1271.

15. Ali NN, Edgar AJ, Samadikuchaksaraei A, Timson CM, Romanska HM, et al. (2002) Derivation of type II alveolar epithelial cells from murine embryonic stem cells. Tissue Eng 8: 541-550.

16. Rippon HJ, Ali NN, Polak JM, Bishop AE (2004) Initial observations on the effect of medium composition on the differentiation of murine embryonic stem cells to alveolar type II cells. Cloning Stem Cells 6: 49-56.

17. Samadikuchaksaraei A, Cohen S, Isaac K, Rippon HJ, Polak JM, et al. (2006) Derivation of distal airway epithelium from human embryonic stem cells. Tissue Eng 12: 867-875.

18. Wang D, Haviland DL, Burns AR, Zsigmond E, Wetsel RA (2007) A pure population of lung alveolar epithelial type II cells derived from human embryonic stem cells. Proc Natl Acad Sci U S A 104: 4449-4454.

19. Banerjee RE (2014) Looking for the elusive lung stem cell niche- A perspective. Translational Respiratory Medicine 2:7-31

20. Banerjee ER, Henderson WR Jr (2013) Role of T cells in a gp91phox knockout murine model of acute allergic asthma. Allergy Asthma Clin Immunol 9: 6.

21. Banerjee ER, Henderson WR Jr (2012) Characterization of lung stem cell niches in a mouse model of bleomycin-induced fibrosis. Stem Cell Res Ther 3: 21 .

22. Banerjee ER, Laflamme MA, Papayannopoulou T, Kahn M, Murry CE, et al. (2012) Human Embryonic Stem Cells Differentiated to Lung LineageSpecific Cells Ameliorate Pulmonary Fibrosis in a Xenograft Transplant Mouse Model. PLoS One 7: e33165:1-15.

23. Banerjee ER, Henderson WR Jr (2012) Defining the molecular role of gp91phox in the immune manifestation of acute allergic asthma using a preclinical murine model. Clin Mol Allergy 10: 2.

24. Banerjee ER (2011) Triple selectin knockout (ELP-/-) mice fail to develop OVA-induced acute asthma phenotype. J Inflamm (Lond) 8: 19.

25. Banerjee ER, Henderson WR Jr (2011) NADPH oxidase has a regulatory role in acute allergic asthma. Journal of Advanced Laboratory Research in Biology 2: 103-120.

26. Banerjee ER, Jiang Y, Henderson WR Jr, Latchman Y, Papayannopoulou $\mathrm{T}$ (2009) Absence of alpha 4 but not beta 2 integrins restrains development of chronic allergic asthma using mouse genetic models. Exp Hematol 37: 715-727.

27. Banerjee ER, Latchman YE, Jiang Y, Priestley GV, Papayannopoulou T (2008) Distinct changes in adult lymphopoiesis in Rag2-/- mice fully reconstituted by alpha4-deficient adult bone marrow cells. Exp Hematol 36: 1004-1013.

28. Inamdar MS, Venu P, Srinivas MS, Rao K, VijayRaghavan K (2009) Derivation and characterization of two sibling human embryonic stem cell lines from discarded grade III embryos. Stem Cells Dev 18: 423-433.
29. Venu P, Chakraborty S, Inamdar MS (2010) Analysis of long-term culture properties and pluripotent character of two sibling human embryonic stem cell lines derived from discarded embryos. In Vitro Cell Dev Biol Anim 46: 200-205.

30. Shetty R, Inamdar MS (2012) Derivation of human embryonic stem cell lines from poor quality embryos. Methods Mol Biol 873: 151-161.

31. Guisbert E, Yura T, Rhodius VA, Gross CA (2008) Convergence of molecular, modeling, and systems approaches for an understanding of the Escherichia coli heat shock response. Microbiol Mol Biol Rev 72: 545-554.

32. Vabules RM, et al. (2010) Cold Spring Harbour Perspectives in Biology.

33. Afzal E, Ebrahimi M, Najafi SM, Daryadel A, Baharvand H (2011) Potential role of heat shock proteins in neural differentiation of murine embryonal carcinoma stem cells (P19). Cell Biol Int 35: 713-720.

34. Chen J, Shi ZD, Ji X, Morales J, Zhang J, et al. (2013) Enhanced osteogenesis of human mesenchymal stem cells by periodic heat shock in self-assembling peptide hydrogel. Tissue Eng Part A 19: 716-728.

35. Chen J, Li C, Wang S (2014) Periodic heat shock accelerated the chondrogenic differentiation of human mesenchymal stem cells in pellet culture. PLoS One 9: e91561.

36. Inamdar MS, Healy L, Sinha A, Stacey G (2012) Global solutions to the challenges of setting up and managing a stem cell laboratory. Stem Cell Rev 8: 830-843.

37. Bonig H, Papayannopoulou T (2012) Mobilization of hematopoietic stem/progenitor cells: general principles and molecular mechanisms. Methods Mol Biol 904: 1-14.

38. Bilican B, Serio A, Barmada SJ, Nishimura AL, Sullivan GJ, et al. (2012) Mutant induced pluripotent stem cell lines recapitulate aspects of TDP-43 proteinopathies and reveal cell-specific vulnerability. Proc Natl Acad Sci U S A 109: 5803-5808.

39. Nestor CE, Ottaviano R, Reddington J, Sproul D, Reinhardt D, et al. (2012) Tissue type is a major modifier of the 5-hydroxymethylcytosine content of human genes. Genome Res 22: 467-477.

40. Barbaric I, Jones M, Buchner K, Baker D, Andrews PW, et al. (2011) Pinacidil enhances survival of cryopreserved human embryonic stem cells. Cryobiology 63: 298-305.

41. Barbaric I, Jones M, Harley DJ, Gokhale PJ, Andrews PW (2011) Highcontent screening for chemical modulators of embryonal carcinoma cell differentiation and survival. J Biomol Screen 16: 603-617.

42. Frost J, Monk D, Moschidou D, Guillot PV, Stanier P, et al. (2011) The effects of culture on genomic imprinting profiles in human embryonic and fetal mesenchymal stem cells. Epigenetics 6: 52-62.

43. Hernandez D, Ruban L, Mason C (2011) Feeder-free culture of human embryonic stem cells for scalable expansion in a reproducible manner. Stem Cells Dev 20: 1089-1098.

44. Lim HJ, Han J, Woo DH, Kim SE, Kim SK, et al. (2011) Biochemical and morphological effects of hypoxic environment on human embryonic stem cells in long-term culture and differentiating embryoid bodies. Mol Cells 31: 123-132.

45. Wright A, Andrews N, Bardsley K, Nielsen JE, Avery K, et al. (2011) Mapping the stem cell state: eight novel human embryonic stem and embryonal carcinoma cell antibodies. Int J Androl 34: e175-187.

46. International Stem Cell Initiative, Amps K, Andrews PW, Anyfantis G, Armstrong L, et al. (2011) Screening a large, ethnically diverse population of human embryonic stem cells identifies a chromosome 20 minimal amplicon that confers a growth advantage. Nat Biotech 29: 1132-1144.

47. Aflatoonian B, Ruban L, Shamsuddin S, Baker D, Andrews P, et al. (2010) Generation of Sheffield (Shef) human embryonic stem cell lines using a microdrop culture system. In Vitro Cell Dev Biol Anim 46: 236-241.

48. Amps KJ, Jones M, Baker D, Moore HD (2010) In situ cryopreservation of human embryonic stem cells in gas-permeable membrane culture cassettes for high post-thaw yield and good manufacturing practice. Cryobiology 60: 344-350.

49. Barbaric I, Gokhale PJ, Jones M, Glen A, Baker D, et al. (2010) Novel regulators of stem cell fates identified by a multivariate phenotype screen 
Citation: Kar S, Hore G, Sanyal S, Banerjee ER (2014) Human Embryonic Stem Cell Lines BJNhem 19 and 20 Fail to Differentiate Into Lung Lineage Specific Cells despite Induction through Guided Endodermal Differentiation. J Tissue Sci Eng 6: 144. doi: $10.4172 / 2157-7552.1000144$

Page 11 of 11

of small compounds on human embryonic stem cell colonies. Stem Cell Res 5: 104-119.

50. Na J, Furue MK, Andrews PW (2010) Inhibition of ERK1/2 prevents neural and mesendodermal differentiation and promotes human embryonic stem cell self-renewal. Stem Cell Res 5: 157-169.

51. Aflatoonian B, Aflatoonian R, et al. (2009) Embryoid bodies from human embryonic stem cells form a niche for male germ cell development in vitro. Papers contributed to the 9th International Congress of Andrology 169-174.

52. Aflatoonian B, Ruban L, Jones M, Aflatoonian R, Fazeli A, et al. (2009) In vitro post-meiotic germ cell development from human embryonic stem cells. Hum Reprod 24: 3150-3159.

53. Kubikova I, Konecna H, Sedo O, Zdrahal Z, Rehulka P, et al. (2009) Proteomic profiling of human embryonic stem cell-derived microvesicles reveals a risk of transfer of proteins of bovine and mouse origin. Cytotherapy 11: 330-340, $1 \mathrm{p}$ following 340.

54. Dean SK, Yulyana Y, Williams G, Sidhu KS, Tuch BE (2006) Differentiation of encapsulated embryonic stem cells after transplantation. Transplantation 82: 1175-1184.

55. Inniss K, Moore H (2006) Mediation of apoptosis and proliferation of human embryonic stem cells by sphingosine-1-phosphate. Stem Cells Dev 15: 789-796.
56. Cerqueira MT, Marques AP, Reis RL (2012) Using stem cells in skin regeneration: possibilities and reality. Stem Cells Dev 21: 1201-1214.

57. Frost J, Monk D, Moschidou D, Guillot PV, Stanier P, et al. (2011) The effects of culture on genomic imprinting profiles in human embryonic and fetal mesenchymal stem cells. Epigenetics 6: 52-62.

58. International Stem Cell Initiative, Amps K, Andrews PW, Anyfantis G Armstrong L, et al. (2011) Screening ethnically diverse human embryonic stem cells identifies a chromosome 20 minimal amplicon conferring growth advantage. Nat Biotechnol 29: 1132-1144.

59. Pringle S, De Bari C, Dell'Accio F, Przyborski S, Cooke MJ, et al. (2011) Mesenchymal differentiation propensity of a human embryonic stem cell line. Cell Prolif 44: 120-127.

60. Karlsson KR, Cowley S, Martinez FO, Shaw M, Minger SL, et al. (2008) Homogeneous monocytes and macrophages from human embryonic stem cells following coculture-free differentiation in M-CSF and IL-3. Exp Hematol 36: 1167-1175.

61. Pickering SJ, Braude PR, Patel M, Burns CJ, Trussler J, et al. (2003) Preimplantation genetic diagnosis as a novel source of embryos for stem cell research. Reprod Biomed Online 7: 353-364.

62. Warburton D, Wuenschell C, Flores-Delgado G, Anderson K (1998) Commitment and differentiation of lung cell lineages. Biochem Cell Biol 76: 971-995. 